\title{
Rosa hybrida extract suppresses vascular smooth muscle cell responses by the targeting of signaling pathways, cell cycle regulation and matrix metalloproteinase-9 expression
}

\author{
SE-JUNG LEE ${ }^{1 *}$, SE YEON WON $^{1 *}$, SUNG LYEA PARK $^{1}$, JUN-HUI SONG $^{1}$, DAE-HWA NOH ${ }^{1}$, \\ HONG-MAN KIM ${ }^{2,3}$, CHANG SHIK YIN ${ }^{3}$, WUN-JAE KIM ${ }^{4}$ and SUNG-KWON MOON ${ }^{1}$ \\ ${ }^{1}$ Department of Food and Nutrition, Chung-Ang University, Anseong-si, Gyeonggi-do 17546; \\ ${ }^{2}$ Graduate School of East-West Medical Science, Kyung Hee University, Seoul 130-701; ${ }^{3}$ Acupuncture Meridian \\ Science Research Center, College of Korean Medicine, Kyung Hee University, Seoul 130-701; ${ }^{4}$ Department of Urology, \\ Chungbuk National University College of Medicine, Cheongju, Chungbuk 361-763, Republic of Korea
}

Received March 5, 2015; Accepted January 29, 2016

DOI: $10.3892 / \mathrm{ijmm} .2016 .2504$

\begin{abstract}
The pharmacological effects of Rosa hybrida are well knownin the cosmeticsindustry. However, the role of Rosahybrida in cardiovascular biology had not previously been investigated, to the best of our knowledge. The aim of the present study was to elucidate the effect of water extract of Rosa hybrida (WERH) on platelet-derived growth factor (PDGF)-stimulated vascular smooth muscle cells (VSMCs). VSMC proliferation, which was stimulated by PDGF, was inhibited in a non-toxic manner by WERH treatment, which also diminished the phosphorylation of extracellular signal-regulated kinase 1/2 (ERK1/2) and AKT. Treatment with WERH also induced G1-phase cell cycle arrest, which was due to the decreased expression of cyclins and cyclin-dependent kinases (CDKs), and induced p21WAF1 expression in PDGF-stimulated VSMCs. Moreover, WERH treatment suppressed the migration and invasion of VSMCs stimulated with PDGF. Treatment with WERH abolished the expression of matrix metalloproteinase-9 (MMP-9) and decreased the binding activity of nuclear factor- $\mathrm{KB}(\mathrm{NF}-\kappa \mathrm{B})$, activator protein-1 (AP-1), and specificity protein 1 (Sp1) motifs in PDGF-stimulated VSMCs. WERH treatment inhibited the proliferation of PDGF-stimulated VSMCs through p21WAF1-mediated G1-phase cell cycle arrest, by decreasing the kinase activity of cyclin/CDK complexes. Furthermore, WERH suppressed the PDGF-induced phosphorylation of ERK1/2 and AKT in VSMCs. Finally, treatment with WERH impeded
\end{abstract}

Correspondence to: Professor Sung-Kwon Moon,Department of Food and Nutrition, Chung-Ang University, Building no. 905, 4726 Seodongdaero, Daedeok-myeon, Anseong-si, Gyeonggi-do 17546, Republic of Korea

E-mail: sumoon66@dreamwiz.com

*Contributed equally

Key words: water extract of Rosa hybrida, vascular smooth muscle cells, platelet-derived growth factor, proliferation, migration, invasion the migration and invasion of VSMCs stimulated by PDGF by downregulating MMP-9 expression and a reduction in NF- $\kappa \mathrm{B}$, AP-1 and Sp1 activity. These results provide new insights into the effects of WERH on PDGF-stimulated VSMCs, and we suggest that WERH has the potential to act as a novel agent for the prevention and/or treatment of vascular diseases.

\section{Introduction}

Vascular smooth muscle cells (VSMCs) play a major role in the morphogenesis of blood vessels (1). Following vascular injury, the rate of VSMC proliferation and migration is greatly increased, as well as the synthetic capacity (1). VSMCs play a role in a number of vascular diseases, including atherosclerosis and restenosis. Abnormal proliferation and migration of VSMCs is pivotal in the development of atherosclerosis (1). Platelet-derived growth factor (PDGF) is known to stimulate the migration and proliferation of VSMCs following injury, which form an intimal vascular lesion $(2,3)$. In addition, PDGF influences the proliferation of VSMCs via the phosphorylation of extracellular signal-regulated kinase 1/2 (ERK1/2), p38 mitogen-activated protein kinase (p38 MAPK), c-Jun $\mathrm{N}$-terminal kinase (JNK), and AKT signaling pathways $(4,5)$.

Cell cycle progression is regulated by cyclin-dependent kinases (CDKs) that are activated by cyclins, which are regulatory subunits, to form cyclin/CDK complexes (6,7). During proliferation, VSMCs initially undergo a G1- to S-phase transition followed by further rounds of proliferation $(1,8)$. The cyclin-dependent kinase inhibitors (CDKIs) p21WAF1 and p27KIP1 negatively control the G1- to S-phase cell cycle progression by inhibiting the kinase activities of cyclin/CDK complexes $(9,10)$. Previous studies have noted the positive regulatory role which p21WAF1 plays in the activation of cyclin/ CDK as an effector of proliferation $(11,12)$.

Matrix metalloproteinase-9 (MMP-9) is a gelatinase that degrades type IV collagen, leading to the migration and invasion of VSMCs, which results in the vascular plaque instability characteristic of vascular diseases, such as atherosclerosis $(13,14)$. Previous research using in vitro and in vivo experiments has 
suggested that the elevated expression of MMP-9 is closely associated with the formation of arterial lesions (14-17). The expression of MMP-9 is markedly induced by growth factors and cytokines although the basal levels of MMP-9 expression are very low (14-17). Previous studies have demonstrated that the induction of MMP-9 expression is strictly regulated at the transcriptional level through the binding activity of nuclear factor $-\kappa \mathrm{B}(\mathrm{NF}-\kappa \mathrm{B})$, specificity protein $1(\mathrm{Sp} 1)$ and activator protein-1 (AP-1) motifs $(18,19)$.

Rosa hybrida is used in the cosmetics industry as a source of aromatic oils (20). Previously, researchers have identified gallic acid and volatiles (1-butanol, dodecyl acrylate and cyclododecane) as the main components of $R$. hybrida (21). Previous studies have established various pharmacological effects for the extract of R. hybrida: it has been noted for its antioxidant, antimicrobial, anti-inflammatory and neuroprotective effects (20-22). However, the inhibitory effect of water extract of Rosa hybrida (WERH) on vascular diseases has not previously been studied to the best of our knowledge. In the present study, we investigated the antiatherogenic role of WERH in regulating proliferation, migration, and invasion in PDGF-stimulated VSMCs.

\section{Materials and methods}

Antibodies. Polyclonal antibodies against cyclin E (sc-481), CDK2 (sc-163) and CDK4 (sc-260), p21WAF1 (sc-756), p53 (sc-126), p27 (sc-528) and GAPDH (sc-20357) were all obtained from Santa Cruz Biotechnology, Inc. (Santa Cruz, CA, USA). Monoclonal antibody against cyclin D1 (04-221) was obtained from Millipore (Temecula, CA, USA). Polyclonal antibodies against ERK (9102), phosphorylated (p)-ERK (9101), p38 MAPK (9212), p-p38 MAPK (9211), JNK (9258), p-JNK (9251), AKT (9272) and p-AKT (9271) were all purchased from Cell Signaling Technology (Lakewood, NJ, USA). Polyclonal MMP-9 antibody (AB19016) was purchased from Millipore. HRP-conjugated secondary antibodies against goat anti-rabbit IgG-HRP (sc-2004), goat anti-mouse IgG-HRP (sc-2005) and donkey anti-goat IgG-HRP (sc-2020) were purchased from Santa Cruz Biotechnology, Inc. All procedures and protocols used in the present study were approved by the Ethics Committee of Chung-Ang University (Anseong, Korea).

Preparation of extract. We used a total of $100 \mathrm{~g}$ of air-dried Rosa Hybrida flowers (purchased from Jincheon Agricultural Technology Center, Jincheon-gun, Korea), which were finely crushed and soaked in water, followed by heating at $100^{\circ} \mathrm{C}$. The extract was concentrated via a rotary evaporator (Rotavapor-R; Brinkmann Instruments, Westbury, NY, USA), lyophilized, and freeze-dried. The yield of the final extract was approximately $10 \% \mathrm{w} / \mathrm{w}$, and the extract was diluted in saline buffer.

Cell cultures. VSMCs were obtained from the aortas of 2 young male Sprague-Dawley rats (8 weeks old, weighing 200-250 g; DHbiolink, Eumseong, Korea) using enzymatic digestion, as described previously (23). Briefly, the rats were sacrificed by $\mathrm{CO}_{2}$ inhalation, the vessels were obtained under sterile conditions and were washed several times in Hanks' balanced salt solution (HBSS). Following removal of the adventitia from the aortas, digestion of the aortas in $5 \mathrm{ml}$ of digestion solution $(0.125 \mathrm{mg} / \mathrm{ml}$ elastase, $0.25 \mathrm{mg} / \mathrm{ml}$ soybean trypsin inhibitor, $10 \mathrm{mg} / \mathrm{ml}$ collagenase I, $2.0 \mathrm{mg} / \mathrm{ml}$ crystallized bovine albumin and $15 \mathrm{mM}$ HEPES; all from Sigma-Aldrich; St. Louis, MO, USA) was performed at $37^{\circ} \mathrm{C}$ for $45 \mathrm{~min}$. The digested cells were filtered with a sterile $100-\mu \mathrm{m}$ nylon mesh (BD Biosciences, Franklin Lakes, NJ, USA), centrifuged at 1,000 rpm for $10 \mathrm{~min}$ and rinsed twice in Dulbecco's modified Eagle's medium (DMEM; Corning, Inc., Corning, NY, USA) containing $10 \%$ fetal calf serum. They were then cultured in DMEM. To characterize the cells as VSMCs, immunofluorescence staining was performed with a monoclonal antibody against smooth muscle- $\alpha$-actin (Sigma-Aldrich). The explants were incubated in DMEM containing 10\% FBS (Corning, Inc.), $2 \mathrm{mM}$ glutamine (Sigma-Aldrich), $50 \mu \mathrm{g} / \mathrm{ml}$ gentamicin (Sigma-Aldrich), and $50 \mu \mathrm{l} / \mathrm{ml}$ amphotericin B (Sigma-Aldrich) at $37^{\circ} \mathrm{C}$ in an atmosphere with $5 \% \mathrm{CO}_{2}$. The cells were used at passages five to eight. For use in the experiments, the cells were grown to $80-90 \%$ confluence and rendered quiescent by serum-starvation for at least $24 \mathrm{~h}$ in DMEM without FBS.

Cell viability assay. The cells were cultured to $80-90 \%$ confluence, and then starved while being cultured in serum-free DMEM for $24 \mathrm{~h}$. The cells were incubated with various concentrations of WERH $(100,200,400$ and $600 \mu \mathrm{g} / \mathrm{ml})$ for $40 \mathrm{~min}$ and then cultured with or without PDGF (20 ng/ml; Millipore) for a further $24 \mathrm{~h}$. A modified 3-(4,5-dimethylthiazol-2-yl)-2,5-diphenyltetrazolium bromide (MTT; Sigma-Aldrich) assay was used to determine cell viability, as described previously $(12,23)$.

Thymidine incorporation assay. After $24 \mathrm{~h}$ starvation, the cells were treated with the indicated concentrations of WERH and then cultured with or without PDGF $(20 \mathrm{ng} / \mathrm{ml})$ for a further $24 \mathrm{~h}$. This was followed by the addition of $1 \mu \mathrm{Ci} / \mathrm{ml}$ [methyl- ${ }^{3} \mathrm{H}$ ] thymidine (PerkinElmer NEN., Boston, MA, USA) for $4 \mathrm{~h}$. Incorporated thymidine was precipitated with cold $10 \%$ trichloroacetic acid, solubilized in $0.2 \mathrm{M} \mathrm{NaOH}$, and counted using a scintillation counter (PerkinElmer NEN, Inc., Waltham, MA, USA).

Cell cycle analysis using fluorescence-activated cell sorting (FACS). The cells were collected and fixed in $70 \%$ ethanol (Millipore) for $2 \mathrm{~h}$ at $4^{\circ} \mathrm{C}$. After washing with ice-cold phosphatebuffered saline (PBS; Sigma-Aldrich), the cells were incubated with RNase ( $0.1 \mathrm{mg} / \mathrm{ml}$; Sigma-Aldrich) and propidium iodide (50 $\mu \mathrm{g} / \mathrm{ml}$; Sigma-Aldrich) for $30 \mathrm{~min}$. Cell cycle analysis was performed using a Becton-Dickinson FACStar flow cytometer equipped with Becton-Dickinson Cell Fit software (both from BD Biosciences), as previously described $(12,23)$.

Immunoblot analysis. The cells were lysed using $250 \mu 1$ lysis buffer [containing, in mmol/1, HEPES (pH 7.5) 50, NaCl 150, EDTA 1, EGTA 2.5, DTT $1, \beta$-glycerophosphate 10 , NaF 1 , $\mathrm{Na}_{3} \mathrm{VO}_{4} 0.1$, and phenylmethylsulfonylfluoride 0.1 and $10 \%$ glycerol, $0.1 \%$ Tween-20, $10 \mu \mathrm{g} / \mathrm{ml}$ of leupeptin, and $2 \mu \mathrm{g} /$ $\mathrm{ml}$ aprotinin; all from Sigma-Andrich]. After centrifugation at $12,000 \mathrm{rpm}$ for $20 \mathrm{~min}$ at $4^{\circ} \mathrm{C}$, the protein concentrations of the cell lysates were determined by Bradford assay. Equal amounts of the samples were resolved via electrophoresis on SDS-polyacrylamide (10\%) gels, and then electroblotted onto nitrocellulose membranes (Hybond; Amersham Corp., Arlington Heights, IL, USA). The membranes were incubated overnight 
with specific primary antibodies at $4^{\circ} \mathrm{C}$ followed by peroxidaseconjugated secondary antibodies for $1 \mathrm{~h}$. Immunocomplexes were visualized using an ECLPlus ${ }^{\mathrm{TM}}$ Western Blot Detection system (Amersham Biosciences, Piscataway NJ, USA). Experiments were performed at least 3 times.

Immunoprecipitation and immune complex kinase assays. For the immunoprecipitation assay, equal amounts of cell lysates were incubated with specific antibodies overnight at $4^{\circ} \mathrm{C}$. Immunocomplexes were added using protein A-sepharose beads (Santa Cruz Biotechnology, Inc.), followed by incubation at $4^{\circ} \mathrm{C}$ for $2 \mathrm{~h}$. The immunoprecipitates were washed with lysis buffer 3 times, and then resuspended in SDS-PAGE sample buffer containing $\beta$-mercaptoethanol (Bio-Rad, Richmond, CA, USA). The samples were analyzed by immunoblot analysis. For immune complex kinase assays, the immunoprecipitated samples were washed 3 times with lysis buffer and twice with kinase buffer (containing, in $\mathrm{mM} / \mathrm{l}$, HEPES 50, $\mathrm{MgCl}_{2} 10$, DTT $1, \beta$-glycerophosphate $10, \mathrm{NaF} 1$, and sodium orthovanadate 0.1 . The pellet was resuspended in $25 \mu \mathrm{l}$ kinase buffer containing either $1 \mu \mathrm{g}$ glutathione S-transferase (GST)-pRb C-terminal (pRb amino acids 769-921 fusion protein; Santa Cruz Biotechnology, Inc.) or $5 \mu \mathrm{g}$ histone $\mathrm{H}_{1}$ (Life Technologies, Grand Island, NY, USA), $20 \mu \mathrm{M} / 1$ ATP, and $5 \mu \mathrm{Ci}\left[\gamma^{32} \mathrm{P}\right] \mathrm{ATP}$ $(4,500 \mu \mathrm{Ci} / \mathrm{mmol}$; ICN, Costa Mesa, CA, USA), and incubated for $20 \mathrm{~min}$ at $30^{\circ} \mathrm{C}$. The reaction was then stopped by the addition of $25 \mu \mathrm{l}$ of $2 \mathrm{X}$ concentrated Laemmli sample buffer (Santa Cruz Biotechnology, Inc.). Electrophoresis was performed with the samples using an SDS-polyacrylamide (10\%) gel. The phosphorylated forms of $\mathrm{pRb}$ and histone $\mathrm{H}_{1}$ were visualized with a BAS 2000 bioimaging analyzer (Fujifilm, Tokyo, Japan).

Wound healing migration assay. Serum-starved cells were grown to $90 \%$ confluence, and then damaged with a pipette tip. The medium was replaced with fresh medium containing different concentrations of WERH in the presence or absence of PDGF $(20 \mathrm{ng} / \mathrm{ml})$. After $24 \mathrm{~h}$ incubation, a phase-contrast microscope (Optika, Ponteranica, Italy) was use to capture images and to evaluate the wound closure effected by cell migration.

Invasion assay. An invasion assay was then performed using modified Boyden chambers with a polycarbonate nucleopore membrane (Corning, Inc.). The serum-starved cells were cultured in DMEM containing WERH with or without PDGF and they were then poured into each of the upper chamber plates for $24 \mathrm{~h}$ to allow for cell invasion through the membrane. Non-invading cells on the upper surface were removed using a cotton swab, and the cells that had invaded the membrane were stained with $0.1 \%$ crystal violet solution (containing $2.5 \mathrm{mM}$ crystal violet, $1 \% \mathrm{MeOH}, 4 \%$ paraformaldehyde) for $20 \mathrm{~min}$. Invasive cells were then quantified by counting the cells, using a microscope, in 6 independent areas at $\times 20$ magnification/well.

Gelatin zymography. Conditioned medium was resolved via electrophoresis of polyacrylamide gels supplemented with $1 \mathrm{mg} / \mathrm{ml}$ gelatin. After washing the gels twice with $2.5 \%$ Triton X-100 at room temperature for $2 \mathrm{~h}$, they were reacted with a buffer $\left(10 \mathrm{mM} \mathrm{CaCl}_{2}, 150 \mathrm{mM} \mathrm{NaCl}\right.$, and $50 \mathrm{mM}$ Tris- $\mathrm{HCl}, \mathrm{pH} \mathrm{7.5)}$ at $37^{\circ} \mathrm{C}$ overnight. The gels were then stained with $0.2 \%$ Coomassie blue, followed by destaining with $50 \%$ methanol and 5\% acetic acid. Areas of gelatinase activity were visualized as clear bands against a dark blue field.

Nuclear extracts and electrophoretic mobility shift assay (EMSA). Nuclear extracts and EMSA were performed as described previously $(12,18)$. The cultured cells were harvested by centrifugation, washed and suspended in a buffer $[10 \mathrm{mM}$ HEPES (pH 7.9), 10 mM KCl, 0.1 mM EDTA, 0.1 mM EGTA, $1 \mathrm{mM}$ DTT and $0.5 \mathrm{mM}$ PMSF] for $15 \mathrm{~min}$ on ice. After vortexing the cells with $0.5 \%$ Nonidet P-40, a nuclear pellet was then assembled by centrifugation at $12,000 \mathrm{rpm}$ for 1-2 min at $4^{\circ} \mathrm{C}$ and extracted in buffer (20 mM HEPES pH 7.9, $0.4 \mathrm{M} \mathrm{NaCl}, 1 \mathrm{mM}$ EDTA, $1 \mathrm{mM}$ EGTA, $1 \mathrm{mM}$ DTT and $1 \mathrm{mM}$ PMSF) for $15 \mathrm{~min}$ at $4^{\circ} \mathrm{C}$. The nuclear extract $(10-20 \mu \mathrm{g})$ was reacted at $4^{\circ} \mathrm{C}$ for 30 min using the 100 -fold excess of an unlabeled oligonucleotide containing the -79 MMP-9 cis element of interest (Bioneer, Daejeon, Korea). The sequences used were as follows: AP-1, CTGACCCCTGAGTCAGCACTT; NF- $\kappa$ B, CAGTGGAATTCCCCAGCC; and Sp1, GCCCAT TCCTTCCGCCCCCAGATGAAGCAG. The binding reaction mixture was then incubated at $4^{\circ} \mathrm{C}$ for $20 \mathrm{~min}$ in a buffer (25 mM HEPES buffer $\mathrm{pH}$ 7.9, 0.5 mM EDTA, $0.5 \mathrm{mM}$ DTT, $0.05 \mathrm{M} \mathrm{NaCl}$, and $2.5 \%$ glycerol) with $2 \mu \mathrm{g}$ poly $(\mathrm{dI} / \mathrm{dC})$ (Bioneer) and $5 \mathrm{fmol}\left(2 \times 10^{4} \mathrm{cpm}\right)$ of a Klenow end-labeled ( ${ }^{32}$ P-ATP) 30-mer oligonucleotide (Bioneer), which spanned the DNA binding site in the MMP-9 promoter. The reaction mixture was electrophoresed at $4{ }^{\circ} \mathrm{C}$ in a $6 \%$ polyacrylamide gel with TBE $(89 \mathrm{mM}$ Tris, $89 \mathrm{mM}$ boric acid and $1 \mathrm{mM}$ EDTA) running buffer. The gel was washed with water, dried and exposed to X-ray film overnight. The shifted bands were detected and evaluated using ImageQuant software (GE Healthcare Life Sciences, Pittsburgh, PA, USA).

Statistical analysis. In the present study, the results are represented as the means \pm SE. Statistical comparisons between groups were analyzed using factorial ANOVA analysis and Fisher's least significant difference test. A P-value $<0.05$ was considered to indicate a statistically significant difference.

\section{Results}

WERH inhibits the PDGF-stimulated proliferation of VSMCs. To evaluate the effect of WERH on cell proliferation, VSMCs were treated with WERH, and this was followed by the addition of PDGF. VMSC proliferation was induced by PDGF, and this was verified by MTT assay and a thymidine incorporation assay. The PDGF-induced proliferative effects were reversed to a control level (non-treatment) at a dose of $400 \mu \mathrm{g} / \mathrm{ml} \mathrm{WERH}$ and cell toxicity was not noted (Fig. 1A and B). Cell death was not observed in the vehicle-treated group (Fig. 1A). The survival of VSMCs was significantly reduced at a $600 \mu \mathrm{g} / \mathrm{ml}$ concentration of WERH (Fig. 1A). Similar results were obtained in a thymidine incorporation experiment (Fig. 1B). Subsequent experiments were performed using a non-toxic cellular concentration of WERH (below $400 \mu \mathrm{g} / \mathrm{ml}$ ). These results demonstrated that WERH inhibited VSMC proliferation induced by PDGF, without causing cell death.

WERH induces G1-phase cell cycle arrest by reducing the expression of cyclins/CDKs in PDGF-stimulated VSMCs. We 

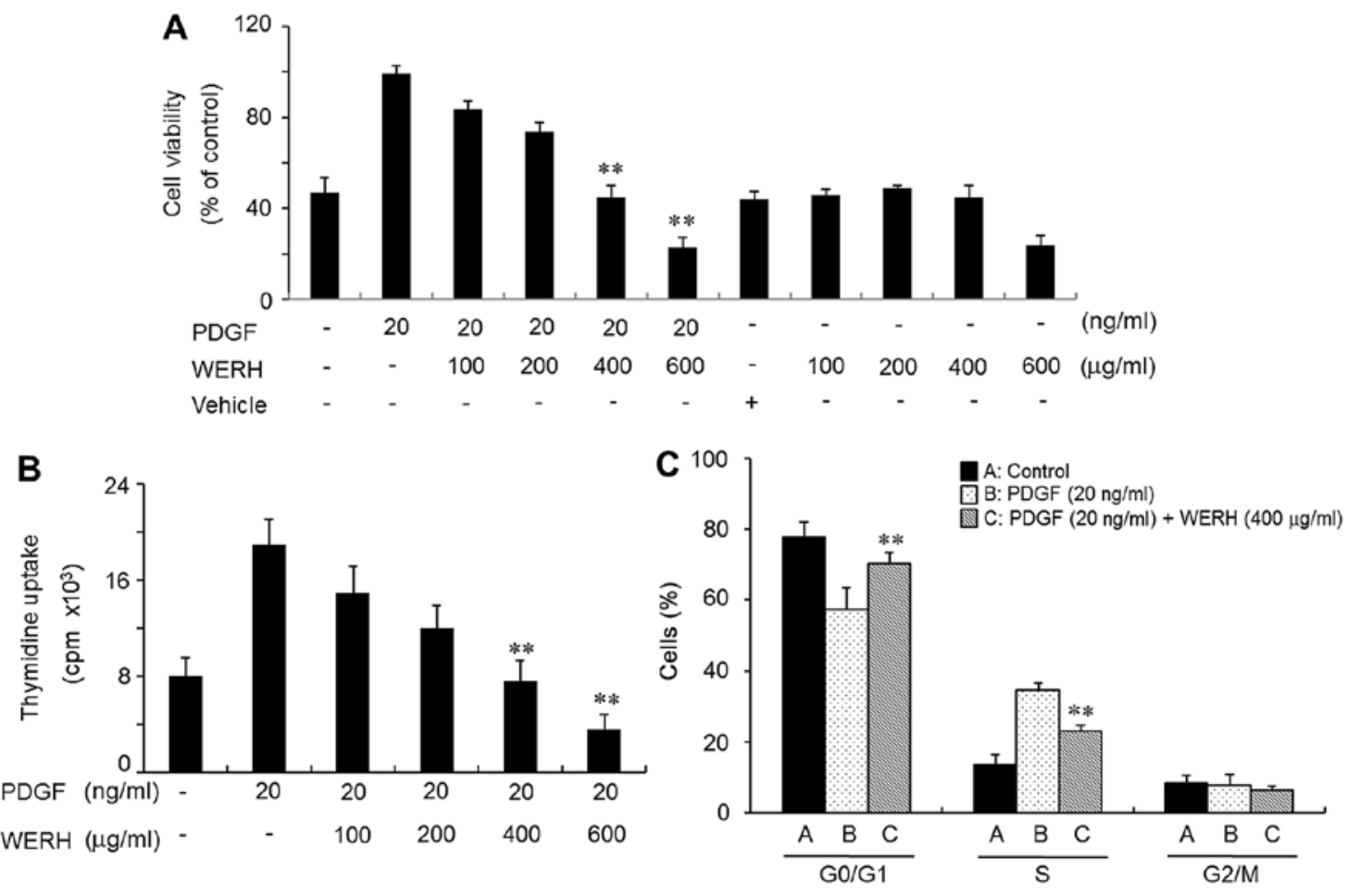

Figure 1. Water extract of Rosa hybrida (WERH) inhibits cell proliferation and induces G1-phase cell-cycle arrest in platelet-derived growth factor (PDGF)-treated vascular smooth muscle cells (VSMCs). After $24 \mathrm{~h}$ serum starvation, VSMCs were exposed to the indicated concentrations of WERH for $40 \mathrm{~min}$, followed by treatment with or without PDGF $(20 \mathrm{ng} / \mathrm{ml})$ for $24 \mathrm{~h}$. PBS was used as the vehicle. (A) An MTT assay was performed to determine cell viability. (B) Cell proliferation was measured by counting thymidine uptake. (C) Cell cycle distribution was evaluated by FACS analysis. All data are expressed as the means $\pm \mathrm{SE}$ of three experiments. ${ }^{* *} \mathrm{P}<0.01$ compared with PDGF stimulation.

then investigated whether WERH induced changes in cell cycle distribution in PDGF-stimulated VSMCs. As shown in Fig. 1C, flow cytometric analysis indicated that in PDGF-stimulated VSMCs WERH treatment induced an accumulation of cells in the G1-phase cell cycle and reduced the number of cells in the S-phase cell cycle, compared with PDGF-stimulated VSMCs which were not treated with WERH. These results suggest that growth inhibition promoted by WERH in VSMCs was caused by G1-phase cell cycle arrest. Subsequent experiments examined the cell cycle-regulatory proteins that are closely associated with the G1-phase cell cycle. The expression levels of cyclin D1, cyclin E, CDK2 and CDK4 were upregulated in PDGF-stimulated VMSCs (Fig. 2A). The treatment of VSMCs with WERH resulted in a significant reduction in the PDGF-stimulated expression levels of cyclin D1, cyclin E, CDK2 and CDK4 (Fig. 2A). The kinase activity of CDKs, as cyclin-CDK complexes, is known to be essential to G1- to $\mathrm{S}$-phase cell cycle progression $(6,7)$. Thus, the following experiment investigated the effect of WERH on the kinase activity of CDKs in PDGF-stimulated VSMCs. PDGF stimulation increased the kinase activity of CDK2 and CDK4 in VSMCs. The increased kinase activity of CDK2 and CDK4 induced by PDGF was suppressed in the presence of WERH (Fig. 2B).

Upregulation of p21WAF1 is associated with WERH-mediated G1-phase cell cycle arrest in PDGF-stimulated VSMCs. As CDKIs are known to be negative regulators that control the transition from the G1- to S-phases of the cell cycle $(9,10)$, the expression levels of the CDKIs p21WAF1 and p27KIP1 were examined. PDGF treatment induced the expression of p21WAF1 and decreased the expression of p27KIP1 in VSMCs (Fig. 2C). Stimulation of VSMCs to PDGF had almost no effect on the expression level of tumor suppressor protein $\mathrm{p} 53$. In addition, WERH treatment increased p21WAF1 expression in PDGF-stimulated VSMCs. However, WERH had no effect on the PDGF-induced inhibition of p27KIP1 expression in VSMCs. The level of p53 expression was not markedly affected by the addition of WERH in the presence of PDGF (Fig. 2C). To further investigate whether the observed inhibitory effect on the CDKs was due to interactions with p21WAF1, immunoprecipitation analysis was performed using cell lysates in the presence of WERH. An increase in the association of p21WAF1 with CDK2 and CDK4 was detected in the PDGF-stimulated VSMCs (Fig. 2D). WERH treatment increased the levels of p21WAF1/CDK2 and p21WAF1/CDK4 in VSMCs following stimulation with PDGF (Fig. 2D). These results demonstrated that WERH suppressed the PDGF-stimulated proliferation of VSMCs, through p21WAF1-mediated G1-phase cell cycle arrest, which involved inhibition of the kinase activity of CDKs.

WERH inhibits the phosphorylation of ERK1/2 and AKT in $P D G F$-treated VSMCs. To identify the signaling pathways involved in the PDGF responses in VSMCs, we examined the phosphorylation of MAPKs and AKT in VSMCs. Stimulation of VSMCs with PDGF led to an increase in the phosphorylation of ERK1/2, JNK, p38 MAPK, and AKT (Fig. 3). We next investigated whether WERH is capable of inhibiting the phosphorylation of ERK1/2, JNK, p38 MAPK and AKT in PDGF-stimulated VSMCs. As shown in Fig. 3, treatment with WERH significantly suppressed the PDGF-induced 

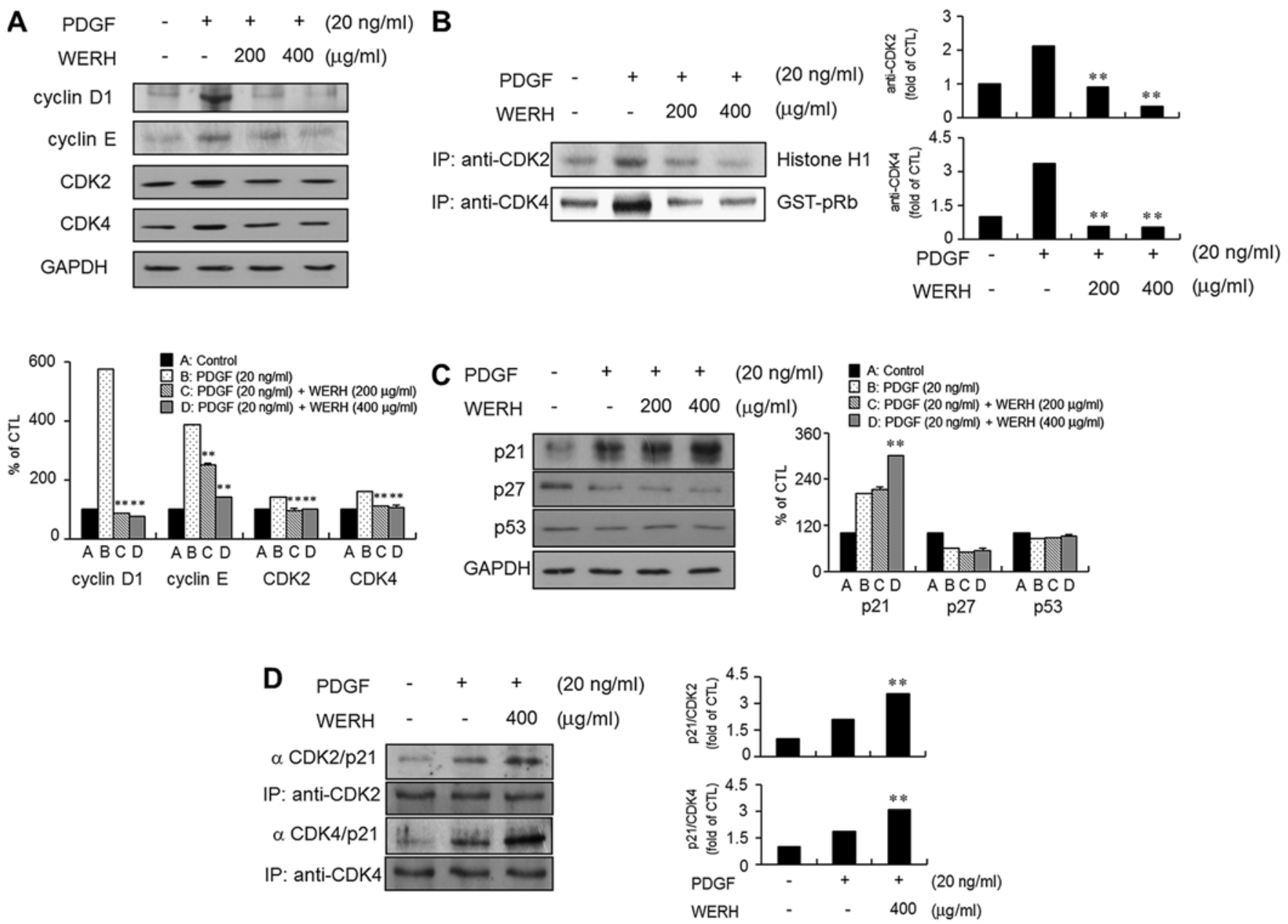

Figure 2. Water extract of Rosa hybrida (WERH) suppresses the expression of cyclins and cyclin-dependent kinases (CDKs) and stimulates expression of p21WAF1 in platelet-derived growth factor (PDGF)-induced vascular smooth muscle cells (VSMCs). Following serum-starvation for $24 \mathrm{~h}$, the cells were pre-incubated with different concentrations of WERH for $40 \mathrm{~min}$ prior to treatment with PDGF $(20 \mathrm{ng} / \mathrm{ml}$ ) and then cultured for $24 \mathrm{~h}$. (A and C) Immunoblot analysis was achieved using antibodies specific for cyclin D1, cyclin E, CDK2, CDK4, p21WAF1, p27KIP1 and p53. The expression levels of each protein were normalized to GAPDH. (B) Total cell lysates from the cells were immunoprecipitated via the addition of anti-CDK2 and anti-CDK4 antibodies. In immunoprecipitates, we analyzed the kinase activation of CDK2 and CDK4 using either histone $\mathrm{H}_{1}$ (for CDK2) or GST-pRb (for CDK4) as the substrate. (D) Following incubation of cell lysates with anti-CDK2 or anti-CDK4 antibodies, immunoblot analysis was performed with the immunoprecipitates using an anti-p21WAF1 antibody. All data are expressed as the means \pm SE of three experiments. ${ }^{* *} \mathrm{P}<0.01$ compared with PDGF stimulation.CTL, control.

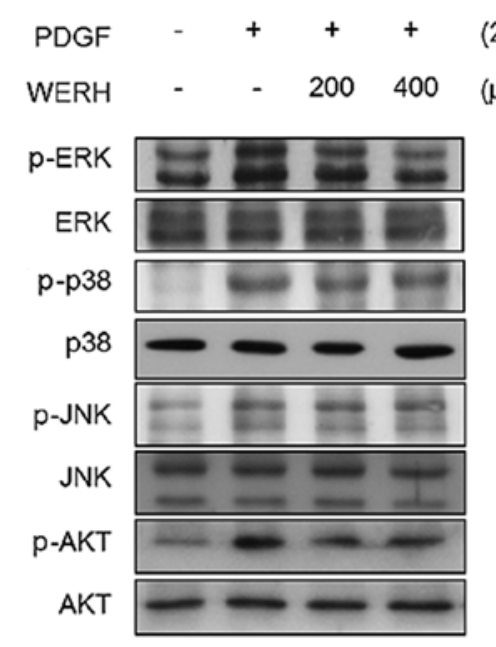

$(20 \mathrm{ng} / \mathrm{ml})$

$(\mu \mathrm{g} / \mathrm{ml})$

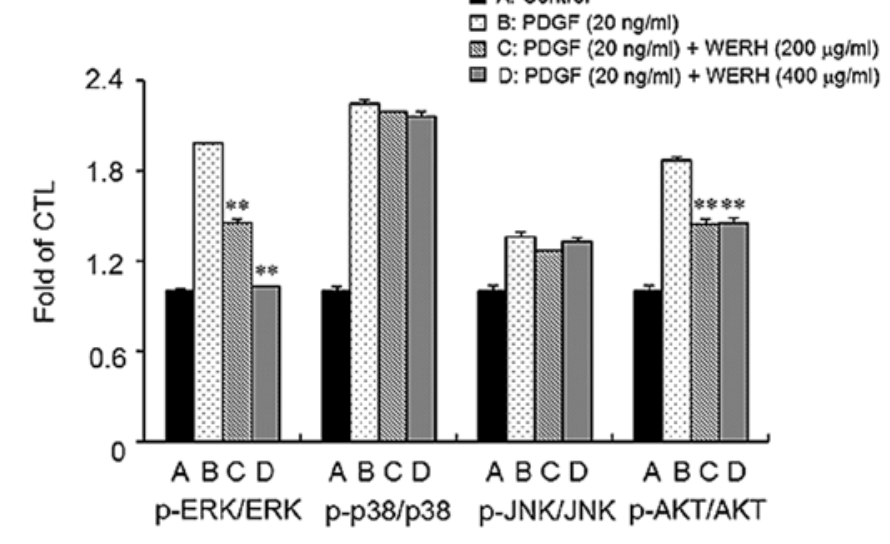

Figure 3. Water extract of Rosa hybrida (WERH) inhibits the phosphorylation of extracellular signal-regulated kinase (ERK)1/2 and AKT in vascular smooth muscle cells (VSMCs) treated with platelet-derived growth factor (PDGF). After serum starvation for $24 \mathrm{~h}$, cells were pretreated with the indicated concentrations of WERH for $40 \mathrm{~min}$, then further incubated with PDGF $(20 \mathrm{ng} / \mathrm{ml})$. Total cell lysates were subjected to immunoblot analysis using antibodies specific for p-ERK1/2, ERK1/2, p-p38, p38, p-c-Jun N-terminal kinase (JNK), JNK, p-AKT and AKT. All data are expressed as the means \pm SE of three experiments. ${ }^{* *} \mathrm{P}<0.01$ compared with PDGF stimulation. 
A Control PDGF (20 ng/ml) PDGF $(20 \mathrm{ng} / \mathrm{ml})+$ WERH $(400 \mu \mathrm{g} / \mathrm{ml})$ WERH $(400 \mu \mathrm{g} / \mathrm{ml})$

$\mathrm{Oh}$
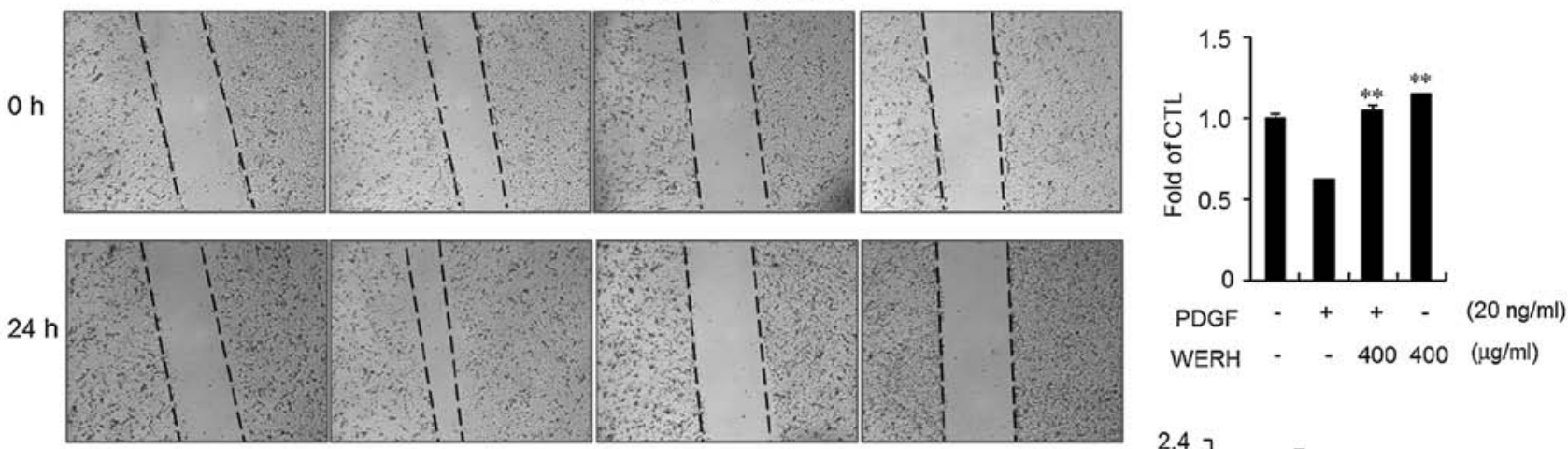

B

Control PDGF $(20 \mathrm{ng} / \mathrm{ml})$

PDGF $(20 \mathrm{ng} / \mathrm{ml})+$
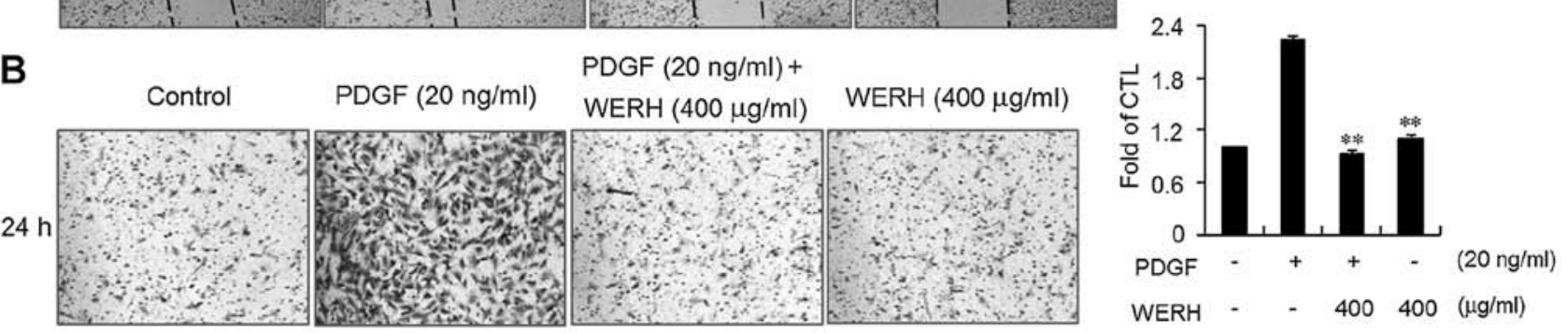

Figure 4. Water extract of Rosa hybrida (WERH) suppresses the migration and invasion of vascular smooth muscle cells (VSMCs) stimulated by platelet-derived growth factor (PDGF). (A) The serum-starved cells were incubated with the indicated concentrations of WERH for 40 min. The cells were scratched with a yellow pipette tip and then incubated with PDGF $(20 \mathrm{ng} / \mathrm{ml})$ for $24 \mathrm{~h}$. The images were captured and evaluated using a microscope. (B) The cells were cultured in serum-starved medium for $24 \mathrm{~h}$. After treatment of cells with the indicated concentrations of WERH, the cells were seeded in Boyden chambers pre-coated with Matrigel. After stimulation with PDGF $(20 \mathrm{ng} / \mathrm{ml})$ for $24 \mathrm{~h}$, the invaded cells were stained with $0.1 \%$ crystal violet solution for $20 \mathrm{~min}$ and photographed with a microscope (magnification, $\mathrm{x} 20$ ). All data are expressed as the means $\pm \mathrm{SE}$ of three experiments. ${ }^{* *} \mathrm{P}<0.01$ compared with PDGF stimulation.

phosphorylation of ERK1/2 and AKT in VSMCs. However, WERH treatment did not markedly affect the phosphorylation of JNK and p38 MAPK induced by PDGF in VSMCs. These results indicated that WERH blocks the proliferation of PDGFstimulated VSMCs by suppressing ERK1/2 and AKT signaling.

WERH suppresses PDGF-induced migration and invasion. The migration and invasion of VSMCs are critical steps in the formation of vascular lesions that lead to the development of atherosclerosis $(13,14)$. An in vitro wound healing assay and an invasion assay were performed to determine the effects of WERH on the PDGF-induced migration and invasion of VSMCs. PDGF treatment significantly increased the motility and invasiveness of VSMCs within $24 \mathrm{~h}$ (Fig. 4). Treatment of VSMCs with WERH resulted in decreased migration of VSMCs (Fig. 4A). In addition, the invasiveness of PDGF-stimulated VSMCs was significantly obstructed in the presence of WERH (Fig. 4B). These results clearly suggest that WERH plays an inhibitory role in the migration and invasion of VSMCs induced by PDGF.

WERH impedes PDGF-induced MMP-9 expression by suppressing $N F-\kappa B, A P-1$ and $S p-1$ binding activities. MMP-9 expression is involved in the migration and invasion of VSMCs during the development of vascular lesions $(13,14)$. Thus, the next experiment examined whether WERH inhibited the expression of MMP-9 in PDGF-stimulated VSMCs using a gelatin zymographic assay and immunoblot analysis. The expression of MMP-9 was stimulated by exposure to PDGF as demonstrated by zymographic assay (Fig. 5A). Treatment with WERH significantly abolished PDGF-stimulated MMP-9 expression in VSMCs. The immunoblot analysis results were similar (Fig. 5A). To further investigate the exact molecular mechanism of action of the inhibitory effect of WERH, an EMSA experiment was employed using three motifs, NF- $\mathrm{KB}$, AP-1 and Sp-1 cis-elements, that are responsible for MMP-9 expression $(18,19)$. PDGF treatment significantly stimulated the binding activity of the NF- $\mathrm{kB}, \mathrm{AP}-1$ and Sp-1 motifs in VSMCs. The addition of WERH markedly reduced the increased binding ability of NF- $\kappa \mathrm{B}, \mathrm{AP}-1$ and Sp-1 motifs in PDGFstimulated VSMCs (Fig. 5B). These results demonstrated that the transcription factors NF- $\mathrm{kB}, \mathrm{AP}-1$ and $\mathrm{Sp}-1$ are involved in the WERH-induced inhibition of MMP-9 expression in PDGFstimulated VSMCs.

\section{Discussion}

The proliferation and migration of mitogen- and growth factor-induced VSMCs is involved in the formation of vascular lesions, which leads to the development of vascular diseases, including atherosclerosis and restenosis (1-3). The role and importance of PDGF in regulating the proliferation and migration of VSMCs has attracted considerable attention $(2,3)$. It is now well established that PDGF, produced by several types of cells, plays a pivotal role as a specific mitogen in VSMCs $(2,3)$. A natural product such as WERH that targets PDGF responses in VSMCs is a potential therapeutic strategy in the prevention or inhibition of vascular diseases. The extract of Rosa hybrida is known to be used in the cosmetics industry (20), and it is also known to exert various pharmacological effects, including antioxidant, anti-inflammatory, antimicrobial and neuroprotective effects (20-22). However, the effect of Rosa hybrida extract 
A $\begin{array}{lcccc}\text { PDGF }(\mathrm{ng} / \mathrm{ml}) & - & 20 & 20 & 20 \\ \text { WERH }(\mu \mathrm{g} / \mathrm{ml}) & - & - & 200 & 400\end{array}$
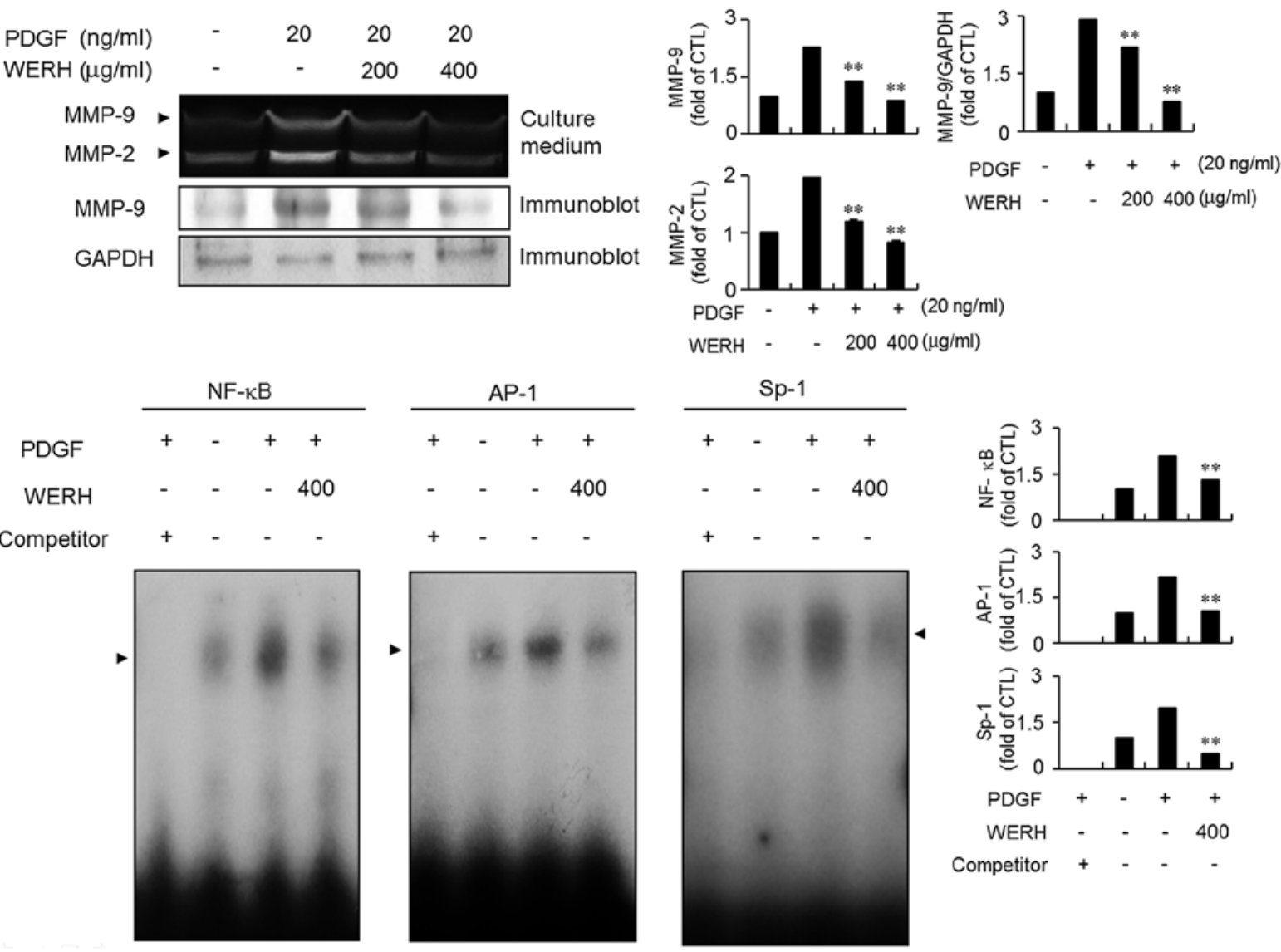

Figure 5. Water extract of Rosa hybrida (WERH) impedes matrix metalloproteinase-9 (MMP-9) expression by decreasing the binding activity of nuclear

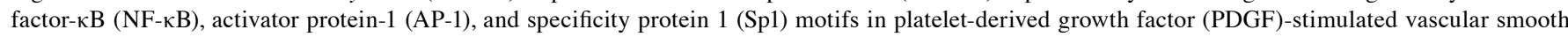
muscle cells (VSMCs). Serum-starved VSMCs were incubated with the indicated concentrations of WERH for 40 min, and then further cultured with PDGF $(20 \mathrm{ng} / \mathrm{ml})$ for $24 \mathrm{~h}$. (A) The expression of MMP-9 was evaluated in conditioned media by zymography. Cell lysates were subjected to immunblot analysis using specific antibodies for MMP-9 and GAPDH. (B) Nuclear extracts were collected from the cells and the activated NF- $\mathrm{KB}$, AP-1 and Sp1 were detected by an electrophoretic mobility shift assay (EMSA) using radiolabeled oligonucleotide probes, respectively. All data are expressed as the means \pm SE of three experiments. ${ }^{* *} \mathrm{P}<0.01$ compared with PDGF stimulation.

on cardiovascular diseases has not yet been examined to the best of our knowledge. In the present study, we investigated the novel suppressive effects of WERH that are involved in the molecular events underlying the proliferation, migration, and invasion of VSMCs in response to PDGF.

One of the key processes that occurs in atherosclerosis is the proliferation of VSMCs stimulated by PDGF (1-3). In the present study, WERH concentrations at and below $400 \mu \mathrm{g} / \mathrm{ml}$ were found to inhibit the proliferation of VSMCs in response to PDGF, in a non-toxic manner. It has previously been pointed out that after vascular injury involving PDGF stimulation, VSMCs proliferate abnormally upon re-entry into the cell cycle $(1,8)$. The findings of the present study showed that WERH induced G1-phase cell cycle arrest in PDGF-stimulated VSMCs. WERH-mediated G1-phase cell cycle arrest was associated with the inhibited expression of cyclins and CDKs. The data also indicated that WERH treatment induced a significant upregulation of p21WAF1 without altering p27KIP1 expression, during G1-phase cell arrest in PDGF-stimulated VSMCs. Taking into consideration the results from the immunoblot analysis demonstrating the increased expression of p21WAF1 in PDGF-stimulated VSMCs, we suggest that p21WAF1 played an important role, as also evidenced by cell proliferation assay. These results clearly demonstrated that the preventive effect of WERH in PDGF-stimulated VSMCs is attributable to p21WAF1-mediated G1-phase cell cycle arrest and inhibition of the kinase activity of cyclin/CDK complexes.

PDGF signaling is involved in the MAPKs and AKT pathways, which are involved in the proliferation of VSMCs $(4,5)$. In the present study, WERH inhibited the PDGF-induced phosphorylation of ERK1/2 in VSMCs. Our results were consistent with those from previous studies demonstrating that phosphorylation of ERK1/2 was inhibited by the hexane fraction of Rosa hybrida in RAW264.7 cells stimulated with lipopolysaccharide (LPS) (24). In addition, we noted that the phosphorylation of AKT was attenuated by the addition of WERH when cells were stimulated with PDGF. This observation is the first evidence of WERH-mediated inhibition of AKT phosphorylation in PDGF-stimulated VSMCs, to the best of our knowledge. However, unexpectedly, treatment with WERH barely affected the phosphorylation of p38 MAPK and JNK signaling in PDGF-stimulated VSMCs. These data demonstrated that a reduction in ERK1/2 phosphorylation and a decrease in AKT phosphorylation occurred when WERH exerted an inhibitory effect on the proliferation of VSMCs stimulated by PDGF.

Migration and invasion of VSMCs are central to vascular lesion formation during atherosclerosis and restenosis (1-3). 
MMPs have been noted to act as important regulators of the migration and invasion of VSMCs (14-17). Notably, enhanced levels of MMP-9 expression have been observed during the formation of arterial lesions in vitro and in vivo (14-17). Previous studies have reported that MMP-9 expression is regulated by transcription factors such as NF- $\kappa \mathrm{B}, \mathrm{Spl}$ and AP-1 motifs $(18,19)$. Thus, we hypothesized that WERH affects the sequential machinery of migration/invasion/MMP-9/transcription factors in PDGF-stimulated VSMCs. Consistent with this hypothesis, our results demonstrated a significant decrease in the wound-healing and invasive ability of PGDF-stimulated VSMCs that had been treated with WERH. Following analysis of the migration and invasion, our data also showed that WERH suppressed the expression of MMP-9 in PDGF-treated VSMCs. Finally, treating VSMCs with WERH significantly inhibited the binding activity of $\mathrm{NF}-\kappa \mathrm{B}, \mathrm{Sp} 1$ and $\mathrm{AP}-1$, which were increased by PDGF in VSMCs. These results demonstrated that WERH treatment abolished PDGF-induced MMP-9 expression in VSMCs by suppressing the binding activity of NF- $\kappa \mathrm{B}$, AP-1 and Sp-1; we hypothesize that this led to an inhibition of the breakdown of ECM and its influence on the migratory and invasive abilities of VSMCs.

The present study elucidates important, novel molecular mechanisms for the effects of WERH in PDGF-stimulated VSMC responses. First, our results suggested that WERH inhibited the proliferation of VSMCs stimulated by PDGF through p21WAF1-mediated G1-phase cell cycle arrest, which was attributed to the reduced kinase activity of cyclin D1/CDK4 and cyclin E/CDK2 complexes. In addition, the treatment of VSMCs with WERH blocked the phosphorylation of ERK1/2 and AKT signaling in response to PDGF. Furthermore, WERH treatment impeded the PDGF-induced migration and invasion of VSMCs, which was regulated through diminished MMP-9 expression, by a decrease in the binding activity of $\mathrm{NF}-\kappa \mathrm{B}$, AP-1 and Sp1 motifs. The findings of the present study suggest that WERH has the potential to be used in the prevention and treatment of atherosclerosis and re-stenosis. Further study is required to identify the active components of WERH and to elucidate its efficacy in animal models.

\section{Acknowledgements}

This study was supported by the Basic Science Research Program through the National Research Foundation of Korea (NRF), funded by the Ministry of Education, Science and Technology (no. 2012R1A6A3A01040720). This study was also supported by the Chung-Ang University Excellent Student Scholarship in 2014.

\section{References}

1. Ross R: Cell biology of atherosclerosis. Annu Rev Physiol 57: 791-804, 1995.

2. Jawien A, Bowen-Pope DF, Lindner V, Schwartz SM and Clowes AW: Platelet-derived growth factor promotes smooth muscle migration and intimal thickening in a rat model of balloon angioplasty. J Clin Invest 89: 507-511, 1992.
3. Ferns GA, Raines EW, Sprugel KH, Motani AS, Reidy MA and Ross R: Inhibition of neointimal smooth muscle accumulation after angioplasty by an antibody to PDGF. Science 253 : 1129-1132, 1991.

4. Owens GK, Kumar MS and Wamhoff BR: Molecular regulation of vascular smooth muscle cell differentiation in development and disease. Physiol Rev 84: 767-801, 2004.

5. Zhan Y, Kim S, Izumi Y, Izumiya Y, Nakao T, Miyazaki H and Iwao H: Role of JNK, p38, and ERK in platelet-derived growth factor-induced vascular proliferation, migration, and gene expression. Arterioscler Thromb Vasc Biol 23: 795-801, 2003.

6. Sherr CJ: G1 phase progression: cycling on cue. Cell 79: 551-555, 1994.

7. Sherr CJ: Cancer cell cycles. Science 274: 1672-1677, 1996.

8. Gordon D, Reidy MA, Benditt EP and Schwartz SM: Cell proliferation in human coronary arteries. Proc Natl Acad Sci USA 87: 4600-4604, 1990.

9. Xiong Y, Hannon GJ, Zhang H, Casso D, Kobayashi R and Beach D: p21 is a universal inhibitor of cyclin kinases. Nature 366: 701-704, 1993.

10. Toyoshima $\mathrm{H}$ and Hunter $\mathrm{T}$ : p27, a novel inhibitor of $\mathrm{G} 1$ cyclin-Cdk protein kinase activity, is related to p21. Cell 78: 67-74, 1994.

11. Weiss RH, Joo A and Randour C: p21(Waf1/Cip1) is an assembly factor required for platelet-derived growth factor-induced vascular smooth muscle cell proliferation. J Biol Chem 275: 10285-10290, 2000.

12. Moon SK, Kim HM, Lee YC and Kim CH: Disialoganglioside (GD3) synthase gene expression suppresses vascular smooth muscle cell responses via the inhibition of ERK1/2 phosphorylation, cell cycle progression, and matrix metalloproteinase-9 expression. J Biol Chem 279: 33063-33070, 2004.

13. Senior RM, Griffin GL, Fliszar CJ, Shapiro SD, Goldberg GI and Welgus HG: Human 92- and 72-kilodalton type IV collagenases are elastases. J Biol Chem 266: 7870-7875, 1991.

14. Newby AC and Zaltsman AB: Molecular mechanisms in intimal hyperplasia. J Pathol 190: 300-309, 2000.

15. Cho A and Reidy MA: Matrix metalloproteinase- 9 is necessary for the regulation of smooth muscle cell replication and migration after arterial injury. Circ Res 91: 845-851, 2002.

16. Galis ZS, Johnson C, Godin D, Magid R, Shipley JM, Senior RM and Ivan E: Targeted disruption of the matrix metalloproteinase-9 gene impairs smooth muscle cell migration and geometrical arterial remodeling. Circ Res 91: 852-859, 2002.

17. Cho A, Graves J and Reidy MA: Mitogen-activated protein kinases mediate matrix metalloproteinase-9 expression in vascular smooth muscle cells. Arterioscler Thromb Vasc Biol 20: 2527-2532, 2000.

18. Moon SK, Cha BY and Kim CH: ERK1/2 mediates TNF-alpha-induced matrix metalloproteinase- 9 expression in human vascular smooth muscle cells via the regulation of NF-kappaB and AP-1: involvement of the ras dependent pathway. J Cell Physiol 198: 417-427, 2004.

19. Bond M, Chase AJ, Baker AH and Newby AC: Inhibition of transcription factor NF-kappaB reduces matrix metalloproteinase-1, -3 and -9 production by vascular smooth muscle cells. Cardiovasc Res 50: 556-565, 2001.

20. Choi EM and Hwang JK: Investigations of anti-inflammatory and antinociceptive activities of Piper cubeba, Physalis angulata and Rosa hybrida. J Ethnopharmacol 89: 171-175, 2003.

21. Yang G, Park D, Lee SH, Bae DK, Yang YH, Kyung J, Kim D, Choi EK, Hong JT, Jeong HS, et al: Neuroprotective effects of a butanol fraction of Rosa hybrida petals in a middle cerebral artery occlusion model. Biomol Ther (Seoul) 21: 454-461, 2013.

22. Lee HR, Lee JM, Choi NS and Lee JM: The antioxidative and antimicrobial ability of ethanol extracts from Rosa hybrida. Korean J Food Sci Technol 35: 373-378, 2003.

23. Lee SJ, Park SS, Kim WJ and Moon SK: Gleditsia sinensis thorn extract inhibits proliferation and TNF- $\alpha$-induced MMP-9 expression in vascular smooth muscle cells. Am J Chin Med 40: 373-386, 2012.

24. Lee HJ, Kim HS, Kim ST, Park D, Hong JT, Kim YB and Joo SS: Anti-inflammatory effects of hexane fraction from white rose flower extracts via inhibition of inflammatory repertoires. Biomol Ther (Seoul) 19: 331-335, 2011. 